Acta Crystallographica Section E

\section{Structure Reports}

\section{Online}

ISSN 1600-5368

\section{R. Allan}

School of Chemistry, The University of Edinburgh, King's Buildings, West Mains Road, Edinburgh EH9 3JJ, Scotland

Correspondence e-mail: d.r.allan@ed.ac.uk

\section{Key indicators}

Single-crystal synchrotron study

$T=205 \mathrm{~K}$

Mean $\sigma(\mathrm{C}-\mathrm{C})=0.003 \AA$

$R$ factor $=0.056$

$w R$ factor $=0.054$

Data-to-parameter ratio $=11.5$

For details of how these key indicators were automatically derived from the article, see http://journals.iucr.org/e.

\title{
Cylopentylamine monohydrate
}

The crystal structure of cylopentylamine monohydrate, $\mathrm{C}_{5} \mathrm{H}_{11} \mathrm{~N} \cdot \mathrm{H}_{2} \mathrm{O}$, is composed of molecular chains of alternating cyclopentylamine and water molecules which are linked by $\mathrm{O}-\mathrm{H} \cdots \mathrm{O}$ and $\mathrm{O}-\mathrm{H} \cdots \mathrm{N}$ hydrogen bonds. These chains are parallel to the monoclinic $b$ axis and they are bridged by weaker $\mathrm{O} \cdots \mathrm{H}-\mathrm{N}$ contacts, forming hydrogen-bonded layers of molecules parallel to (100).

\section{Comment}

The crystal structure of cylopentylamine monohydrate, (I), was determined at $205 \mathrm{~K}$ (just below the $\sim 215 \mathrm{~K}$ melting point) as part of a series of studies on the structural behaviour of prototypical hydrogen-bonded molecular systems at conditions of either non-ambient temperature or pressure. It crystallizes in the monoclinic space group $P 2_{1} / c$ with one cyclopentylamine molecule and a single water molecule in the asymmetric unit (Fig. 1).

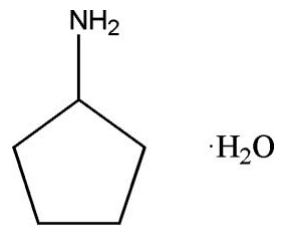

(I)

The water molecules are linked by $\mathrm{O}-\mathrm{H} \cdots \mathrm{O}$ hydrogen bonds, forming the backbone of molecular chains which run parallel to the $b$ axis, while $\mathrm{O}-\mathrm{H} \cdots \mathrm{N}$ hydrogen bonds link the cyclopentylamine molecules to this backbone in an alternating sequence (Fig. 2 and Table 1). The lengths of these hydrogen bonds are fairly similar, while the weaker $\mathrm{O}-\mathrm{H} \cdots \mathrm{N}$ hydrogen bond is correspondingly less linear. Significantly weaker $\mathrm{O} \cdots \mathrm{H}-\mathrm{N}$ contacts bridge neighbouring molecular chains, forming slabs of molecules parallel to (100) (Fig. 3). One of these $\mathrm{O} \cdots \mathrm{N}$ distances is marginal.

\section{Experimental}

The sample of cyclopentylamine monohydrate was prepared from anhydrous starting material (of $99 \%$ purity, as received from Aldrich) and placed in a sealed glass capillary tube with an internal diameter of ca $0.2 \mathrm{~mm}$. The sample was cooled using an Oxford Cryosystems lowtemperature device (Cosier \& Glazer, 1986) until crystallization was observed. The temperature was then cycled between 180 and $215 \mathrm{~K}$, and the capillary successively translated through the gas stream, so that the sample was partially remelted and the number of crystallites reduced. The final sample, at $205 \mathrm{~K}$, was composed of a small number
Received 25 January 2006 Accepted 13 February 2006
(C) 2006 International Union of Crystallography All rights reserved 


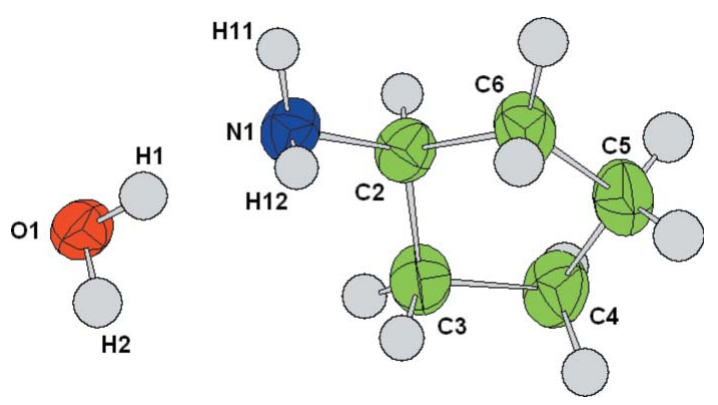

Figure 1

The asymmetric unit of (I), showing $30 \%$ probability displacement ellipsoids.

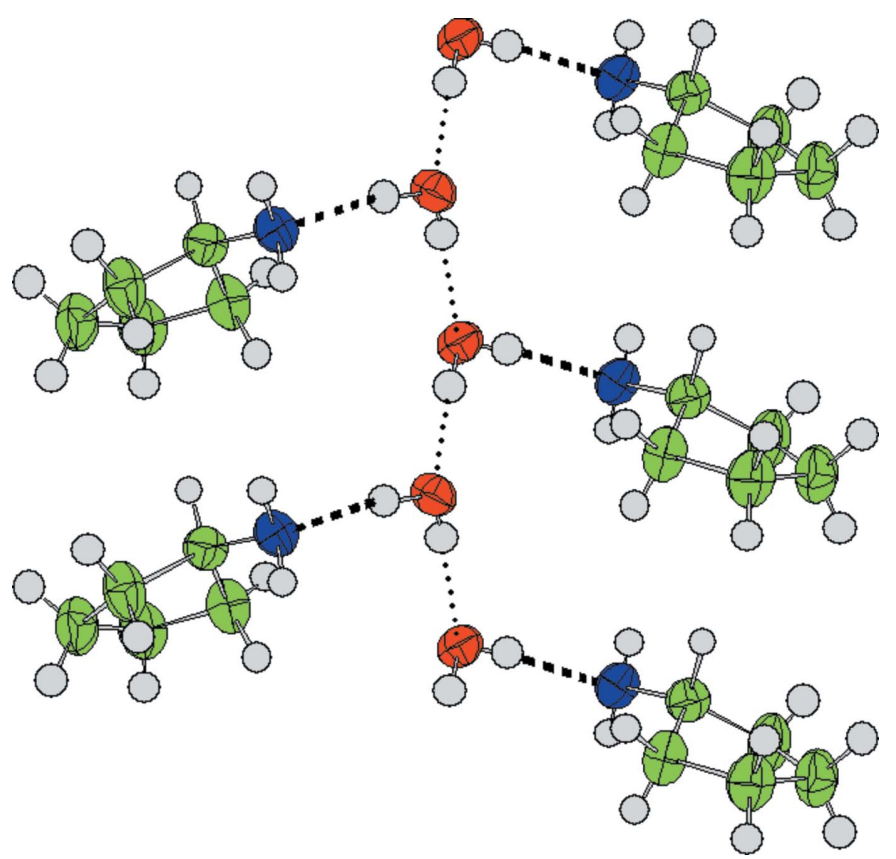

Figure 2

The hydrogen-bonded molecular chains of (I), viewed perpendicular to (101). The $\mathrm{O}-\mathrm{H} \cdots \mathrm{O}$ and $\mathrm{O}-\mathrm{H} \cdots \mathrm{N}$ hydrogen bonds are shown as light dotted lines and heavy dashed lines, respectively.

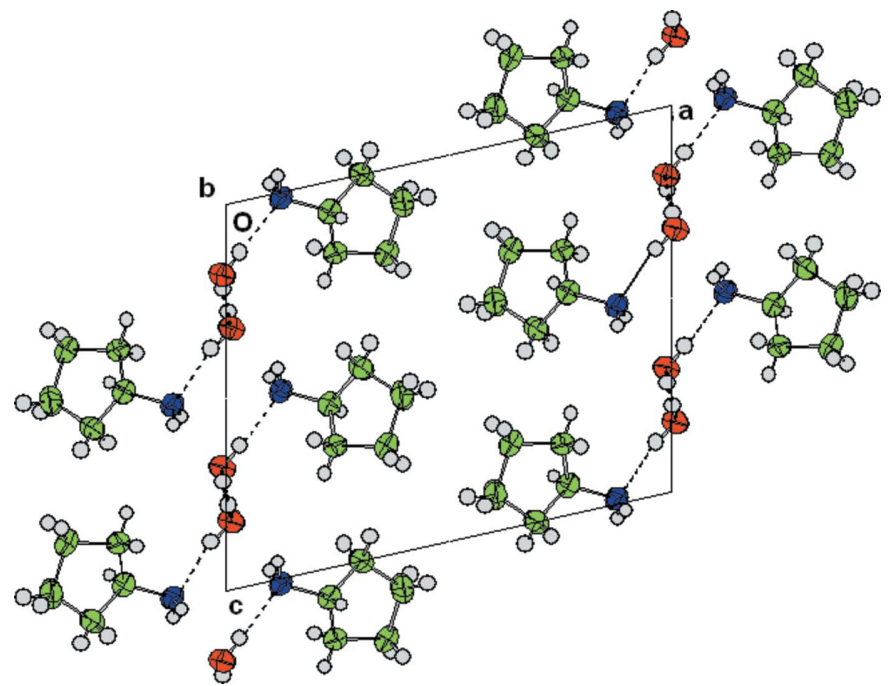

Figure 3

The packing of (I), viewed along the $b$ axis. The $\mathrm{O}-\mathrm{H} \cdots \mathrm{O}$ and $\mathrm{O}-$ $\mathrm{H} \cdots \mathrm{N}$ hydrogen bonds are shown as dashed lines. of crystals and the reflections from the largest of these were indexed and their intensities subsequently used for structure solution.
Crystal data

$\mathrm{C}_{5} \mathrm{H}_{11} \mathrm{~N} \cdot \mathrm{H}_{2} \mathrm{O}$

$M_{r}=103.16$

Monoclinic, $P 2_{b} / c$

$a=12.969(4) \AA$

$b=4.7125(13) \AA$

$c=11.005(3) \AA$

$\beta=102.614(17)^{\circ}$

$V=656.4(3) \AA^{3}$

$Z=4$

$D_{x}=1.044 \mathrm{Mg} \mathrm{m}^{-3}$

\section{Data collection}

Bruker SMART diffractometer $\varphi$ and $\omega$ scans

Absorption correction: multi-scan (SADABS; Sheldrick, 2004)

$T_{\min }=0.35, T_{\max }=0.99$

5211 measured reflections

1573 independent reflections

Refinement

Refinement on $F$

$R\left[F^{2}>2 \sigma\left(F^{2}\right)\right]=0.056$

$w R\left(F^{2}\right)=0.054$

$S=1.13$

875 reflections

76 parameters

$\mathrm{H}$ atoms treated by a mixture of independent and constrained refinement
Synchrotron radiation

$\lambda=0.6813 \AA$

Cell parameters from 445 reflections

$\theta=8-43^{\circ}$

$\mu=0.07 \mathrm{~mm}^{-1}$

$T=205 \mathrm{~K}$

Cylinder, colourless

$0.20 \times 0.10$ (radius) $\mathrm{mm}$

875 reflections with $I>2 \sigma(I)$

$R_{\text {int }}=0.051$

$\theta_{\max }=27.5^{\circ}$

$h=-17 \rightarrow 17$

$k=-6 \rightarrow 6$

$l=-14 \rightarrow 14$

Modified Chebychev polynomial (Watkin, 1994; Prince, 1982) with the coefficients $2.88,-1.06,1.90$ $(\Delta / \sigma)_{\max }=0.001$

$\Delta \rho_{\max }=0.25{\mathrm{e} \AA^{-3}}^{-3}$

$\Delta \rho_{\min }=-0.16$ e $\AA^{-3}$
Table 1

Hydrogen-bond geometry $\left(\AA{ }^{\circ}\right)$.

\begin{tabular}{|c|c|c|c|c|}
\hline$D-\mathrm{H} \cdots A$ & $D-\mathrm{H}$ & $\mathrm{H} \cdots A$ & $D \cdots A$ & $D-\mathrm{H} \cdots A$ \\
\hline $\mathrm{O} 1-\mathrm{H} 1 \cdots \mathrm{N} 1$ & $0.82(1)$ & $2.01(1)$ & $2.821(2)$ & $172(2)$ \\
\hline $\mathrm{O} 1-\mathrm{H} 2 \cdots \mathrm{O} 1^{\mathrm{i}}$ & $0.82(1)$ & $2.00(1)$ & $2.820(1)$ & $178(3)$ \\
\hline $\mathrm{N} 1-\mathrm{H} 11 \ldots \mathrm{O} 1^{\mathrm{ii}}$ & 0.89 (1) & 2.35 (1) & $3.137(2)$ & $148(2)$ \\
\hline $\mathrm{N} 1-\mathrm{H} 12 \cdots \mathrm{O} 1^{\mathrm{iii}}$ & 0.89 (1) & $2.55(1)$ & $3.426(2)$ & $166(2)$ \\
\hline
\end{tabular}

$\mathrm{H}$ atoms attached to $\mathrm{C}$ atoms were placed in idealized positions $(\mathrm{C}-\mathrm{H}=0.96-1.00 \AA)$ and allowed to ride on their parent atoms. $\mathrm{H}$ atoms attached to $\mathrm{N}$ and $\mathrm{O}$ atoms were located in a difference map and restrained to idealized distances and angles $[\mathrm{N}-\mathrm{H}=0.90$ (1) $\AA$, $\mathrm{O}-\mathrm{H}=0.82(1) \AA$ and $\left.\mathrm{O}-\mathrm{H}-\mathrm{O}=104(1)^{\circ}\right]$. All $\mathrm{H}$ atoms were constrained so that $U_{\text {iso }}(\mathrm{H})$ values were equal to $1.2 U_{\text {eq }}$ of their respective parent atoms.

Data collection: SMART (Bruker, 2001); cell refinement: SAINT; data reduction: SAINT (Bruker, 2003); program(s) used to solve structure: SIR92 (Altomare et al., 1994); program(s) used to refine structure: CRYSTALS (Betteridge et al., 2003); molecular graphics: CAMERON (Watkin et al., 1996); software used to prepare material for publication: CRYSTALS and PLATON (Spek, 2003).

We thank Dr T. Prior of Daresbury Laboratory for his help during the experiment on station 9.8 at SRS. We also thank the EPSRC for funding both this project and DRA's Advanced Research Fellowship. 


\section{organic papers}

\section{References}

Altomare, A., Burla, M. C., Camalli, G., Cascarano, G., Giacovazzo, C., Guagliardi, A. \& Polidori, G. (1994). J. Appl. Cryst. 27, 435-436.

Betteridge, P. W., Carruthers, J. R., Cooper, R. I., Prout, K. \& Watkin, D. J. (2003). J. Appl. Cryst. 36, 1487.

Bruker (2001). SMART. Bruker AXS Inc., Madison, Wisconsin, USA.

Bruker (2003). SAINT. Bruker AXS Inc., Madison, Wisconsin, USA.
Cosier, J. \& Glazer, A. M. (1986). J. Appl. Cryst. 19, 105-107.

Prince, E. (1982). Mathematical Techniques in Crystallography and Materials Science. New York: Springer-Verlag.

Sheldrick, G. M. (2004). SADABS. University of Gottingen, Germany.

Spek, A. L. (2003). J. Appl. Cryst. 36, 7-13.

Watkin, D. J. (1994). Acta Cryst. A50, 411-437.

Watkin, D. J., Prout, C. K. \& Pearce, L. (1996). CAMERON. Chemical Crystallography Laboratory, University of Oxford, England. 


\title{
supporting information
}

Acta Cryst. (2006). E62, o1064-o1066 [https://doi.org/10.1107/S1600536806005198]

\section{Cylopentylamine monohydrate}

\author{
D. R. Allan
}

Cylopentylamine monohydrate

Crystal data

$\mathrm{C}_{5} \mathrm{H}_{11} \mathrm{~N} \cdot \mathrm{H}_{2} \mathrm{O}$

$M_{r}=103.16$

Monoclinic, $P 2_{1} / c$

Hall symbol: $-\mathrm{P} 2 \mathrm{ybc}$

$a=12.969(4) \AA$

$b=4.7125(13) \AA$

$c=11.005(3) \AA$

$\beta=102.614(17)^{\circ}$

$V=656.4(3) \AA^{3}$

$Z=4$

$F(000)=232$

$D_{\mathrm{x}}=1.044 \mathrm{Mg} \mathrm{m}^{-3}$

Synchrotron radiation, $\lambda=0.68130 \AA$

Cell parameters from 445 reflections

$\theta=8-43^{\circ}$

$\mu=0.07 \mathrm{~mm}^{-1}$

$T=205 \mathrm{~K}$

Cylinder, colourless

$0.20 \times 0.10 \times 0.10 \times 0.10$ (radius) $\mathrm{mm}$

\section{Data collection}

Bruker SMART

diffractometer

Curved silicon monochromator

$\varphi$ and $\omega$ scans

Absorption correction: multi-scan

(SADABS; Sheldrick, 2004)

$T_{\text {min }}=0.35, T_{\max }=0.99$

5211 measured reflections

1573 independent reflections

875 reflections with $I>2 \sigma(I)$

$R_{\text {int }}=0.051$

$\theta_{\max }=27.5^{\circ}, \theta_{\min }=4.3^{\circ}$

$h=-17 \rightarrow 17$

$k=-6 \rightarrow 6$

$l=-14 \rightarrow 14$

\section{Refinement}

Refinement on $F$

Least-squares matrix: full

$R\left[F^{2}>2 \sigma\left(F^{2}\right)\right]=0.056$

$w R\left(F^{2}\right)=0.054$

$S=1.13$

875 reflections

76 parameters

5 restraints

Primary atom site location: structure-invariant direct methods

Hydrogen site location: inferred from neighbouring sites

$\mathrm{H}$ atoms treated by a mixture of independent and constrained refinement

Modified Chebychev polynomial (Watkin, 1994; Prince, 1982) with the coefficients 2.88 ,

$-1.06,1.90$

$(\Delta / \sigma)_{\max }=0.001$

$\Delta \rho_{\max }=0.25 \mathrm{e} \AA^{-3}$

$\Delta \rho_{\min }=-0.16$ e $\AA^{-3}$

Fractional atomic coordinates and isotropic or equivalent isotropic displacement parameters $\left(\AA^{2}\right)$

\begin{tabular}{lllll}
\hline & $x$ & $y$ & $z$ & $U_{\text {iso }} * / U_{\text {eq }}$ \\
\hline O1 & $1.01039(11)$ & $0.1644(3)$ & $0.82203(12)$ & 0.0497 \\
N1 & $0.87797(12)$ & $0.2930(4)$ & $0.98670(14)$ & 0.0473 \\
C2 & $0.76853(14)$ & $0.3412(4)$ & $0.92430(17)$ & 0.0473 \\
C3 & $0.75593(15)$ & $0.5310(5)$ & $0.81296(17)$ & 0.0552
\end{tabular}




$\begin{array}{lllll}\text { C6 } & 0.69733(15) & 0.4823(6) & 1.00014(19) & 0.0638 \\ \text { C4 } & 0.63976(15) & 0.6122(6) & 0.78280(19) & 0.0630 \\ \text { C5 } & 0.60449(16) & 0.5968(6) & 0.9053(2) & 0.0678 \\ \text { H21 } & 0.7390 & 0.1534 & 0.8947 & 0.0574^{*} \\ \text { H32 } & 0.7998 & 0.7044 & 0.8354 & 0.0677^{*} \\ \text { H31 } & 0.7771 & 0.4371 & 0.7433 & 0.0675^{*} \\ \text { H62 } & 0.7337 & 0.6375 & 1.0504 & 0.0798^{*} \\ \text { H61 } & 0.6744 & 0.3489 & 1.0547 & 0.0796^{*} \\ \text { H42 } & 0.6295 & 0.8019 & 0.7461 & 0.0731^{*} \\ \text { H41 } & 0.5999 & 0.4774 & 0.7238 & 0.0730^{*} \\ \text { H52 } & 0.5840 & 0.7785 & 0.9321 & 0.0824^{*} \\ \text { H51 } & 0.5454 & 0.4708 & 0.9009 & 0.0827^{*} \\ \text { H1 } & 0.9678(15) & 0.188(5) & 0.8667(18) & 0.0777^{*} \\ \text { H12 } & 0.9016(16) & 0.454(3) & 1.0256(19) & 0.0630^{*} \\ \text { H2 } & 1.0062(19) & 0.311(3) & 0.7810(19) & 0.0780^{*} \\ \text { H11 } & 0.8823(17) & 0.155(4) & 1.0422(16) & 0.0627^{*}\end{array}$

Atomic displacement parameters $\left(\AA^{2}\right)$

\begin{tabular}{lllllll}
\hline & $U^{11}$ & $U^{22}$ & $U^{33}$ & $U^{12}$ & $U^{13}$ & $U^{23}$ \\
\hline O1 & $0.0579(8)$ & $0.0455(8)$ & $0.0482(8)$ & $0.0092(7)$ & $0.0172(6)$ & $0.0043(6)$ \\
N1 & $0.0446(9)$ & $0.0530(11)$ & $0.0420(8)$ & $0.0072(8)$ & $0.0044(7)$ & $0.0023(8)$ \\
C2 & $0.0442(10)$ & $0.0479(11)$ & $0.0474(10)$ & $0.0012(9)$ & $0.0046(8)$ & $-0.0010(9)$ \\
C3 & $0.0486(10)$ & $0.0762(15)$ & $0.0403(10)$ & $0.0097(11)$ & $0.0089(8)$ & $0.0055(10)$ \\
C6 & $0.0542(12)$ & $0.0909(17)$ & $0.0500(11)$ & $0.0145(12)$ & $0.0194(9)$ & $0.0110(12)$ \\
C4 & $0.0465(11)$ & $0.0831(17)$ & $0.0536(12)$ & $0.0070(11)$ & $-0.0021(9)$ & $0.0056(11)$ \\
C5 & $0.0438(11)$ & $0.0847(17)$ & $0.0756(15)$ & $0.0073(11)$ & $0.0148(10)$ & $0.0102(13)$
\end{tabular}

Geometric parameters $\left(\AA,{ }^{\circ}\right)$

\begin{tabular}{llll}
\hline $\mathrm{O} 1-\mathrm{H} 1$ & $0.823(9)$ & $\mathrm{C} 3-\mathrm{H} 31$ & 0.975 \\
$\mathrm{O} 1-\mathrm{H} 2$ & $0.822(9)$ & $\mathrm{C} 6-\mathrm{C} 5$ & $1.510(3)$ \\
$\mathrm{N} 1-\mathrm{C} 2$ & $1.453(2)$ & $\mathrm{C} 6-\mathrm{H} 62$ & 0.975 \\
$\mathrm{~N} 1-\mathrm{H} 12$ & $0.894(9)$ & $\mathrm{C} 6-\mathrm{H} 61$ & 0.960 \\
$\mathrm{~N} 1-\mathrm{H} 11$ & $0.887(9)$ & $\mathrm{C} 4-\mathrm{C} 5$ & $1.517(3)$ \\
$\mathrm{C} 2-\mathrm{C} 3$ & $1.497(3)$ & $\mathrm{C} 4-\mathrm{H} 42$ & 0.978 \\
$\mathrm{C} 2-\mathrm{C} 6$ & $1.526(3)$ & $\mathrm{C} 4-\mathrm{H} 41$ & 0.973 \\
$\mathrm{C} 2-\mathrm{H} 21$ & 0.991 & $\mathrm{C} 5-\mathrm{H} 52$ & 0.961 \\
$\mathrm{C} 3-\mathrm{C} 4$ & $1.519(3)$ & $\mathrm{C} 5-\mathrm{H} 51$ & 0.962 \\
$\mathrm{C} 3-\mathrm{H} 32$ & 0.995 & & \\
& & & \\
$\mathrm{H} 1-\mathrm{O} 1-\mathrm{H} 2$ & $103.9(9)$ & $\mathrm{C} 2-\mathrm{C} 6-\mathrm{H} 62$ & 111.3 \\
$\mathrm{C} 2-\mathrm{N} 1-\mathrm{H} 12$ & $106.9(14)$ & $\mathrm{C} 5-\mathrm{C} 6-\mathrm{H} 62$ & 111.4 \\
$\mathrm{C} 2-\mathrm{N} 1-\mathrm{H} 11$ & $110.4(14)$ & $\mathrm{C} 5-\mathrm{C} 6-\mathrm{H} 61$ & 110.9 \\
$\mathrm{H} 12-\mathrm{H} 1-\mathrm{H} 11$ & $109.4(19)$ & $\mathrm{H} 62-\mathrm{C} 6-\mathrm{H} 61$ & 108.3 \\
$\mathrm{~N} 1-\mathrm{C} 2-\mathrm{C} 3$ & $113.65(16)$ & $\mathrm{C} 3-\mathrm{C} 4-\mathrm{C} 5$ & $105.72(16)$ \\
$\mathrm{N} 1-\mathrm{C} 2-\mathrm{C} 6$ & $117.06(16)$ & $\mathrm{C} 3-\mathrm{C} 4-\mathrm{H} 42$ & 111.0 \\
$\mathrm{C} 3-\mathrm{C} 2-\mathrm{C} 6$ & $102.50(17)$ & &
\end{tabular}




$\begin{array}{llll}\mathrm{N} 1-\mathrm{C} 2-\mathrm{H} 21 & 106.4 & \mathrm{C} 5-\mathrm{C} 4-\mathrm{H} 42 & 111.8 \\ \mathrm{C} 3-\mathrm{C} 2-\mathrm{H} 21 & 107.4 & \mathrm{C} 3-\mathrm{C} 4-\mathrm{H} 41 & 109.8 \\ \mathrm{C} 6-\mathrm{C} 2-\mathrm{H} 21 & 109.4 & \mathrm{C} 5-\mathrm{C} 4-\mathrm{H} 41 & 110.0 \\ \mathrm{C} 2-\mathrm{C} 3-\mathrm{C} 4 & 104.77(17) & \mathrm{H} 42-\mathrm{C} 4-\mathrm{H} 41 & 108.4 \\ \mathrm{C} 2-\mathrm{C} 3-\mathrm{H} 32 & 109.5 & \mathrm{C} 4-\mathrm{C} 5-\mathrm{C} 6 & 106.34(16) \\ \mathrm{C} 4-\mathrm{C} 3-\mathrm{H} 32 & 109.5 & \mathrm{C} 4-\mathrm{C} 5-\mathrm{H} 52 & 112.7 \\ \mathrm{C} 2-\mathrm{C} 3-\mathrm{H} 31 & 111.9 & \mathrm{C} 6-\mathrm{C} 5-\mathrm{H} 52 & 109.8 \\ \mathrm{C} 4-\mathrm{C} 3-\mathrm{H} 31 & 112.3 & \mathrm{C} 4-\mathrm{C} 5-\mathrm{H} 51 & 112.1 \\ \mathrm{H} 32-\mathrm{C} 3-\mathrm{H} 31 & 108.9 & \mathrm{C} 6-\mathrm{C} 5-\mathrm{H} 51 & 108.7 \\ \mathrm{C} 2-\mathrm{C} 6-\mathrm{C} 5 & 105.32(17) & \mathrm{H} 52-\mathrm{C} 5-\mathrm{H} 51 & 107.2\end{array}$

Hydrogen-bond geometry $\left(\AA,{ }^{\circ}\right)$

\begin{tabular}{lllll}
\hline$D-\mathrm{H} \cdots A$ & $D-\mathrm{H}$ & $\mathrm{H} \cdots A$ & $D \cdots A$ & $D-\mathrm{H} \cdots A$ \\
\hline $\mathrm{O} 1-\mathrm{H} 1 \cdots \mathrm{N} 1$ & $0.82(1)$ & $2.01(1)$ & $2.821(2)$ & $172(2)$ \\
$\mathrm{O} 1-\mathrm{H} 2 \cdots \mathrm{O} 1^{\mathrm{i}}$ & $0.82(1)$ & $2.00(1)$ & $2.820(1)$ & $178(3)$ \\
$\mathrm{N} 1-\mathrm{H} 11 \cdots \mathrm{O} 1^{\mathrm{ii}}$ & $0.89(1)$ & $2.35(1)$ & $3.137(2)$ & $148(2)$ \\
$\mathrm{N} 1-\mathrm{H} 12 \cdots \mathrm{O} 1^{\mathrm{iii}}$ & $0.89(1)$ & $2.55(1)$ & $3.426(2)$ & $166(2)$
\end{tabular}

Symmetry codes: (i) $-x+2, y+1 / 2,-z+3 / 2$; (ii) $-x+2,-y,-z+2$; (iii) $-x+2,-y+1,-z+2$. 